\title{
An Explanation for Observed Flux Creep in Opposite Direction to Lorentz Force in Partially-Magnetized Bulk Superconductors
}

\author{
Mark D. Ainslie, Senior Member, IEEE, Roy Weinstein, and Ravi-Persad Sawh
}

\begin{abstract}
Sawh et al. recently reported experimental results that showed, in the case of a partially-magnetized bulk superconductor, magnetized using zero-field-cooling, that flux creep resulted in a reduced field measured at the center of the top surface of the bulk. The authors reported that this may suggest magnetic flux vortices, in this case, move against the Lorentz force, contravening commonly-accepted theory. In this paper, we report the results of numerical simulations explaining the observed measurements, and show that the vortices do indeed move with the Lorentz force, but that geometric effects from the finite geometry of the bulk and the form of the resulting induced supercurrent flowing within the bulk play a key role in this observed phenomenon. As a result, the relaxation of the magnetic flux can result in a measured magnetic field above the bulk superconductor that could be perceived as magnetic flux moving against the Lorentz force, when applying a simple Bean model (infinite slab) analysis to the problem.
\end{abstract}

Index Terms-Bulk high-temperature superconductors, finite element method, numerical simulation, trapped field magnets, Lorentz force.

\section{INTRODUCTION}

T HE Bean model [1], [2] has severe limitations when analyzing the magnetization of bulk superconductors, including geometric effects, the in-field and location dependence of $J_{\mathrm{c}}$, and in the case of pulsed field magnetization (PFM), thermal effects due to the heat generated from the rapid dynamic movement of magnetic flux vortices within the sample, as well as the temperature-dependence of $J_{\mathrm{c}}[3]$. Sawh et al. recently reported experimental results that showed, in the case of a partially-magnetized bulk superconductor, magnetized using zero-field-cooling (ZFC), flux creep resulted in a reduced field measured at the center of the top surface of the bulk [4]. The authors reported that, when applying a simple Bean model (infinite slab) analysis, this may suggest magnetic flux vortices, in this case, move against the Lorentz force, contravening commonly-accepted theory.

In this paper, a 2D axisymmetric numerical model based on the finite element method and implementing the $\boldsymbol{H}$-formulation

Manuscript receipt and acceptance dates will be inserted here. The work of M. D. Ainslie was supported by the Engineering and Physical Sciences Research Council Early Career Fellowship EP/P020313/1. (Corresponding author: Mark. D Ainslie.)

M. D. Ainslie is with the Bulk Superconductivity Group, Department of Engineering, University of Cambridge, Cambridge CB2 1PZ, U.K. (e-mail: mark.ainslie@eng.cam.ac.uk). is used to explain the observed measurements. It is shown that the vortices do indeed move with the Lorentz force, but that geometric effects from the finite geometry of the bulk and the form of the resulting induced supercurrent flowing within the bulk play a key role in this observed phenomenon.

\section{NUMERICAL SIMULATION FRAMEWORK}

The numerical model used in this paper has been used previously to simulate bulk high-temperature superconducting (HTS) materials under various magnetization conditions [5][9], as well as field-cooling (FC) magnetization of $\mathrm{MgB}_{2}$ [10] and iron-pnictide [11] bulks. The governing equations for the electromagnetic properties of the of the bulk superconductor are derived from Maxwell's equations - namely, Faraday's (1) and Ampere's (2) laws:

$$
\begin{gathered}
\nabla \times \boldsymbol{E}+\left(\frac{d \boldsymbol{B}}{d t}\right)=\nabla \times \boldsymbol{E}+\frac{d\left(\mu_{0} \mu_{\mathrm{r}} \boldsymbol{H}\right)}{d t}=0 \\
\nabla \times \boldsymbol{H}=\boldsymbol{J}
\end{gathered}
$$

where $\boldsymbol{H}=\left[H_{\mathrm{r}}, H_{\mathrm{z}}\right]$ represents the magnetic field components, $\boldsymbol{J}=\left[J_{\varphi}\right]$ represents the current density and $\boldsymbol{E}=\left[E_{\varphi}\right]$ represents the electric field. $\mu_{0}$ is the permeability of free space and the relative permeability, $\mu_{\mathrm{r}}$, is 1 . The $E-J$ power law $\left(E \alpha J^{\mathrm{m}}\right)$ [12], [13] is used to simulate the non-linear electrical resistivity of the superconductor, where $n$ defines the steepness of the transition between the superconducting state and normal state. A finite $n$ value also accounts for flux creep relaxation, which results in logarithmic decay of the trapped field after the magnetizing field is removed [14]. For HTS materials, like the one under investigation here, $n$ usually varies between 5 (strong flux creep) and 50 (limiting value between HTS and low-temperature superconducting (LTS) materials), and $n>20$ is a good approximation of Bean's critical state model [3]; hence, in this work, $n=20$ is assumed. The characteristic voltage criterion $E_{0}$ $=1 \times 10^{-4} \mathrm{~V} / \mathrm{m}$ is also assumed. In order to simplify the analysis, a constant $J_{\mathrm{c}}$ approximation is used, where $J_{\mathrm{c}}=2.4 \times 10^{8} \mathrm{~A} / \mathrm{m}^{2}$.

R. Weinstein is with the Texas Center for Superconductivity and Physics Department, University of Houston, Houston TX, United States. R. Weinstein and R-P. Sawh are with Roxxyquest Magnetics, Houston TX, United States.

Color versions of one or more of the figures in this paper are available online at http://ieeexplore.ieee.org.

Digital Object Identifier will be inserted here upon acceptance. 
This is based on one of the samples investigated in detail in [4], for which a surface trapped field $B_{\mathrm{t}, \max }=1.088 \mathrm{~T}$ at $77 \mathrm{~K}$ was reported for a $20 \mathrm{~mm}$ diameter, $8 \mathrm{~mm}$ thick single-grain, topseeded melt-textured bulk Y-Ba-Cu-O sample, when fully magnetized. By setting appropriate boundary conditions, a uniform background magnetizing field is ramped from 0 to $5 \mathrm{~T}$ (a magnetic field large enough to fully magnetize the sample) at 25 $\mathrm{mT} / \mathrm{s}(\Delta t=200 \mathrm{~s})$, then ramped back to $0 \mathrm{~T}$ at the same rate, resulting in a simulated trapped field of approximately $1 \mathrm{~T}$ at the center of the top surface of the bulk. Finally, isothermal conditions are assumed because the ZFC magnetization process is slow; hence, no thermal model is included and a constant temperature $T_{0}=77 \mathrm{~K}$ is assumed.

\section{NUMERICAL SimUlation RESUltS}

For the reader's reference, Fig. 1 shows the original observed magnetic field distribution at $t=3 \mathrm{~min}$ (circles) and $t=1003$ min (squares) across the surface of the $B_{\mathrm{t}, \max }=1.088 \mathrm{~T}$ sample in [4], partially magnetized by an applied field $B_{\mathrm{A}}=0.840 \mathrm{~T}$. Also included is the effect of flux creep, i.e., $B_{\mathrm{t}}(1003 \mathrm{~min})-$ $B_{\mathrm{t}}(3 \mathrm{~min})$, which is indicated by the triangles. Fig. 2 then shows the results of the numerical simulation in the same manner: the magnetic field distribution above the top surface of the bulk, at $z=+0.8 \mathrm{~mm}$ (the same location as the scanning Hall probe in [4]), at $t=+3$ min and $t=+13$ min after partial magnetization, as well as the effect of flux creep, $B_{\mathrm{t}}(+13 \mathrm{~min})-B_{\mathrm{t}}(+3 \mathrm{~min})$. It should be noted that, in the interest of reducing the computational time required, the model was run until $t=+13 \mathrm{~min}$, rather than the experimental $t=+1003 \mathrm{~min}$. There is excellent qualitative and quantitative agreement between the experimental results and the numerical simulation, with subtle differences that may be attributed to the simplistic assumptions made in the model, which neglects any in-field or location dependence of $J_{\text {c }}$. In particular, the numerical model reproduces the experimentally observed result in [4]: a reduced field at the center of the top surface of the bulk when the flux creeps after partial magnetization.

The numerical model provides a powerful tool to understand the physical mechanism of this result by calculating the current density and Lorentz force distributions within the bulk, which are not easily accessible experimentally. Fig. 3 shows the current density distribution within the bulk at three distinct points of time: (a) $t=200 \mathrm{~s}$, at the peak of the applied field in the ZFC process, (b) $t=+3 \mathrm{~min}$ after activation, i.e., $t=580 \mathrm{~s}$, and (c) $t$ $=+13 \mathrm{~min}$ after activation, i.e., $t=1780 \mathrm{~s}$. There are two opposing supercurrent loops flowing within the bulk in this partially-magnetized case, which is expected. However, because of the finite geometry of the bulk, the current distribution deviates from that which would be expected when applying a simple Bean model (infinite slab) analysis, such that the supercurrent flows nearer the center of the bulk towards the top and bottom surfaces. The impact of flux creep on this current density distribution is such that the current decays back towards the periphery of the bulk, resulting in a reduced magnetic field at $r=0$ above the top surface.

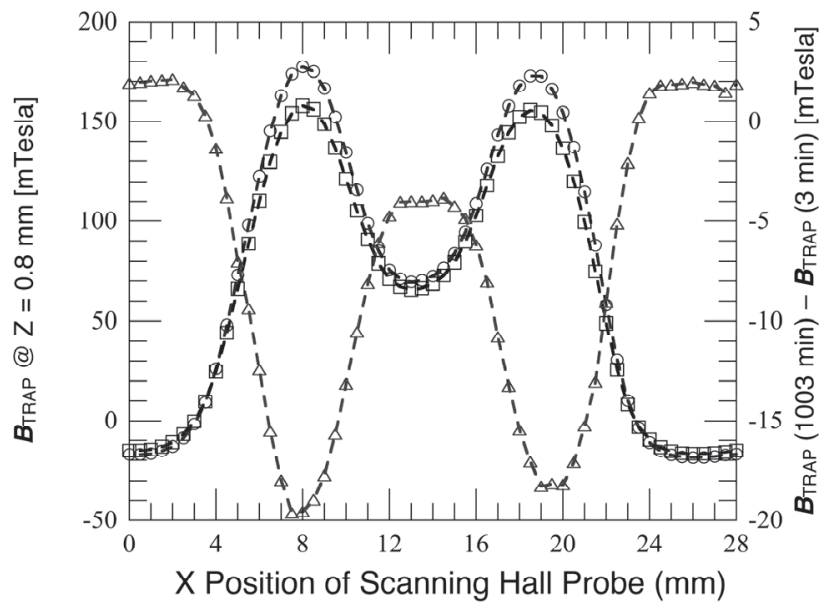

Fig. 1. Original observed magnetic field distribution at $t=3 \mathrm{~min}$ (circles) and $t$ $=1003 \mathrm{~min}$ (squares) across the surface of the $B_{\mathrm{t}, \max }=1.088 \mathrm{~T}$ sample in [4] at $z=+0.8 \mathrm{~mm}$, partially magnetized by an applied field $B_{\mathrm{A}}=0.840 \mathrm{~T}$. Also included is the effect of flux creep, i.e., $B_{\mathrm{t}}(1003 \mathrm{~min})-B_{\mathrm{t}}(3 \mathrm{~min})$, which is indicated by the triangles. Reproduced from [4]. C IOP Publishing Ltd. All rights reserved.

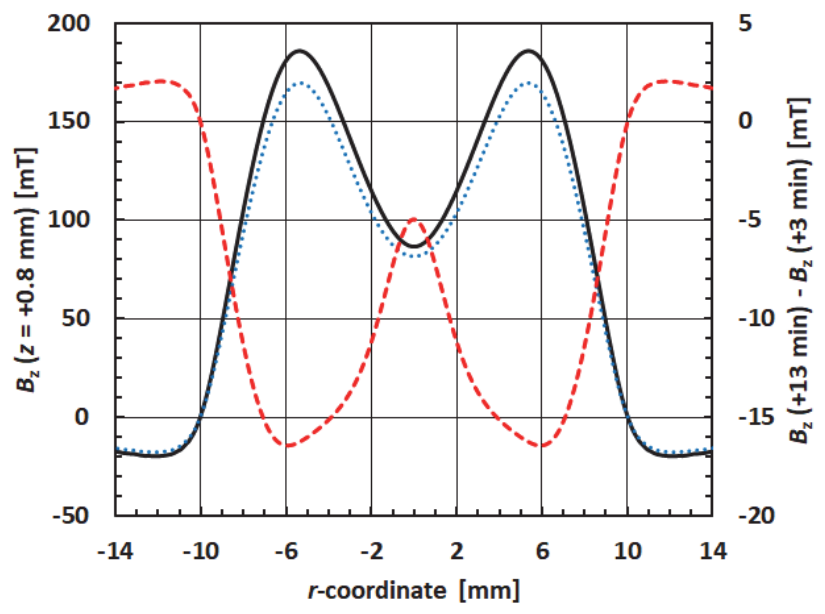

Fig. 2. Simulated magnetic field distribution above the top surface of the bulk, at $z=+0.8 \mathrm{~mm}$, at $t=+3 \mathrm{~min}$ (solid line) and $t=+13 \mathrm{~min}$ (dotted line) after partial magnetization by an applied field $B_{\mathrm{A}}=0.840 \mathrm{~T}$, as well as the effect of flux creep, $B_{\mathrm{t}}(+13 \mathrm{~min})-B_{\mathrm{t}}(+3 \mathrm{~min})$ (red, dashed line).

Fig. 4 shows the Lorentz force, $\boldsymbol{F}_{\mathbf{L}}=\boldsymbol{J} \times \boldsymbol{B}$, distribution within the bulk at $t=200 \mathrm{~s}$ (the peak of the applied field), with arrows included to more clearly indicate the direction of the Lorentz force, and Fig. 5 shows the same plot for (a) $t=+3 \mathrm{~min}$ after activation ( $t=580 \mathrm{~s}$ ) and (b) $t=+13$ min after activation $(t=1780 \mathrm{~s})$. Across the midpoint of the bulk along the thickness, the Lorentz force is directed towards $r=0$ for the inner circulating current loop and directed towards the periphery of the bulk for the outer circulating current loop, as expected from the Bean model and explained in [4]. However, closer to the top and bottom surfaces, it can be seen that this is not true. The direction of the Lorentz force in these locations is towards the center of the bulk along the thickness, which results in decay of the current in this direction, as seen in Fig. 3. 

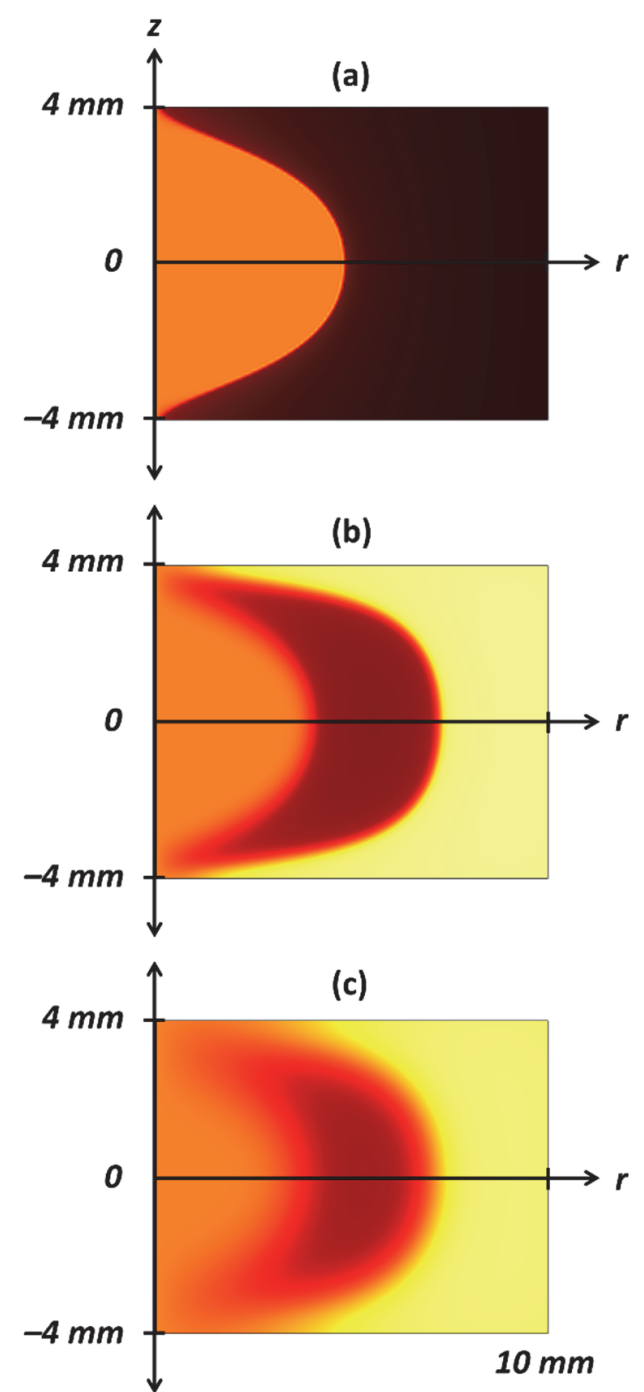

Fig. 3. Simulated current density distribution within the bulk at three distinct points of time: (a) $t=200 \mathrm{~s}$, at the peak of the applied field in the ZFC process, (b) $t=+3 \mathrm{~min}$ after activation, i.e., $t=580 \mathrm{~s}$, and (c) $t=+13 \mathrm{~min}$ after activation, i.e., $t=1780 \mathrm{~s}$.

Finally, Fig. 6 shows the current density across the midpoint of the bulk $(z=0 \mathrm{~mm})$ and $0.2 \mathrm{~mm}$ below the top surface $(z=$ $3.8 \mathrm{~mm})$ at $t=+3 \mathrm{~min}$ and $t=+13 \mathrm{~min}$ after activation, which reinforces the results shown in Figs. 3 and 4: the current density distribution nearer the top and bottom surfaces decays back into the bulk, towards the periphery, and the location of the Hall sensor in magnetization measurements is closest to this location. On the other hand, the current density distribution towards the midpoint of the bulk along the thickness decays towards $r=0$, as expected from the Bean model.

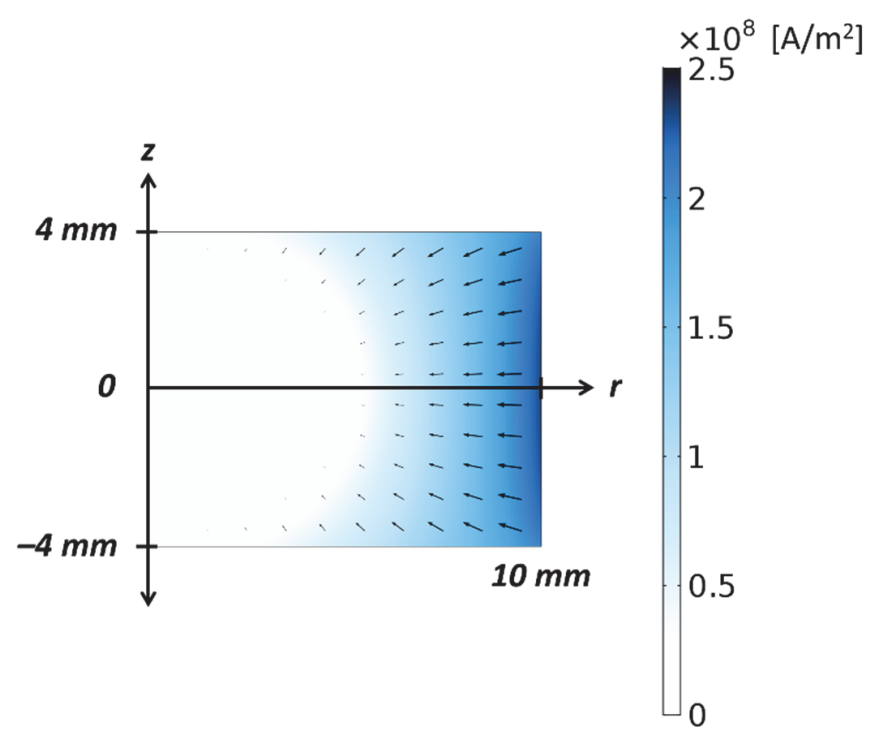

Fig. 4. Lorentz force, $\boldsymbol{F}_{\mathbf{L}}=\boldsymbol{J} \times \boldsymbol{B}$, distribution within the bulk at $t=200 \mathrm{~s}$ (the peak of the applied field). Arrows are included to more clearly indicate the direction of the Lorentz force.

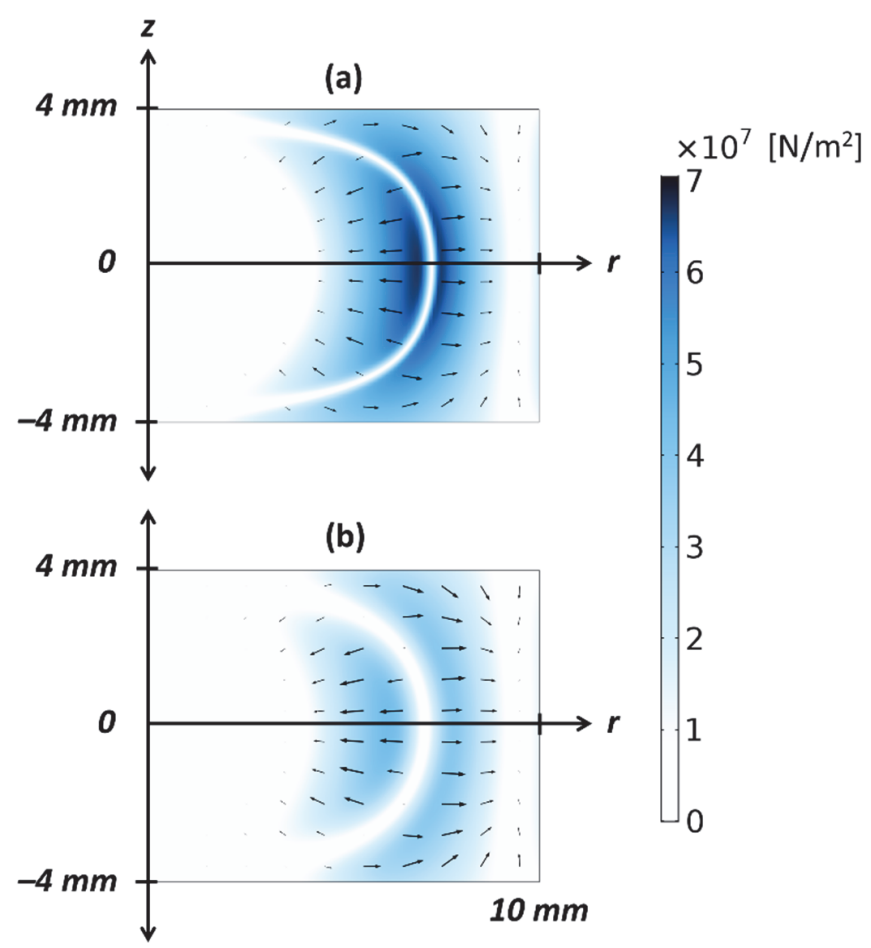

Fig. 5. Lorentz force, $\boldsymbol{F}_{\mathbf{L}}=\boldsymbol{J} \times \boldsymbol{B}$, distribution within the bulk at (a) $t=+3 \mathrm{~min}$ after activation $(t=580 \mathrm{~s})$ and (b) $t=+13 \mathrm{~min}$ after activation $(t=1780 \mathrm{~s})$. Arrows are included to more clearly indicate the direction of the Lorentz force. 


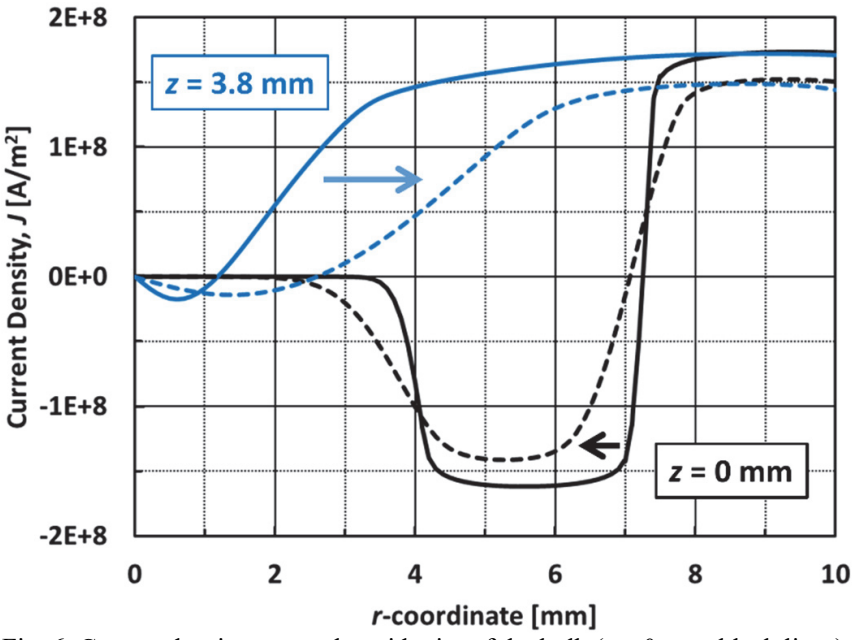

Fig. 6. Current density across the midpoint of the bulk $(z=0 \mathrm{~mm}$, black lines $)$ and $0.2 \mathrm{~mm}$ below the top surface $(z=3.8 \mathrm{~mm}$, blue lines $)$ at $t=+3$ min after activation ( $t=580 \mathrm{~s}$, solid lines) and $t=+13 \mathrm{~min}$ after activation $(t=1780 \mathrm{~s}$, dashed lines). The arrows indicate the direction of the flux creep.

\section{CONCLUSION}

In this paper, a 2D axisymmetric model based on the finite element method and implementing the $\boldsymbol{H}$-formulation is used to explain recently reported experimental results that showed, in the case of a partially-magnetized bulk superconductor, magnetized using zero-field-cooling, that flux creep resulted in a reduced field measured at the center of the top surface of the bulk. The numerical results show that geometric effects from the finite geometry of the bulk and the form of the resulting induced supercurrent play a key role in this observed phenomenon, which deviates from what one may expect when applying a simple Bean model (infinite slab) analysis to the problem.

\section{ACKNOWLEDGMENT}

All data are provided in full in the results section of this paper.

\section{REFERENCES}

[1] C. P. Bean, "Magnetization of Hard Superconductors," Phys. Rev. Lett. vol. 8 , no. 6 , pp. 250-253, 1962.

[2] C. P. Bean, "Magnetization of High-Field Superconductors," Rev. Mod. Phys., vol. 36, no. 1, pp. 31-39, 1964.

[3] M. D. Ainslie and H. Fujishiro, "Modelling of bulk superconductor magnetization," Supercond. Sci. Technol., vol. 28, no. 5, 2015, Art. ID 053002.

[4] R.-P. Sawh, R. Weinstein, K. Carpenter, D. Parks, and K. Davey, "Observation of flux-creep in direction opposite to the Lorentz force," Supercond. Sci. Technol., vol. 30, no. 4, 2017, Art. ID 045006.

[5] J. Zou, M. D. Ainslie, D. Hu, and D. A. Cardwell, "Influence of TimeVarying External Magnetic Fields on Trapped Fields in Bulk Superconductors," IEEE Trans. Appl. Supercond., vol. 25, no. 3, Jun. 2015, Art. ID 4900505.

[6] M. P. Philippe et al., "Influence of soft ferromagnetic sections on the magnetic flux density profile of a large grain, bulk Y-Ba-Cu-O superconductor," Supercond. Sci. Technol., vol. 28, no. 9, 2015, Art. ID 095008.

[7] J. Zou, M. D. Ainslie, D. Hu, and D. A. Cardwell, "Mitigation of Demagnetization of Bulk Superconductors by Time-Varying External Magnetic Fields," IEEE Trans. Appl. Supercond., vol. 26, no. 4, Jun. 2016, Art. ID 8200605 .
[8] M. D. Ainslie et al., "Enhanced trapped field performance of bulk hightemperature superconductors using split coil, pulsed field magnetization with an iron yoke," Supercond. Sci. Technol., vol. 29, no. 7, 2016, Art. ID 074003 .

[9] M. D. Ainslie et al., "Flux jump-assisted pulsed field magnetisation of high- $J_{\mathrm{c}}$ bulk high-temperature superconductors," Supercond. Sci. Technol., vol. 29, no. 12, 2016, Art. ID 124004

[10] J. Zou et al., "Numerical modelling and comparison of $\mathrm{MgB}_{2}$ bulks fabricated by HIP and infiltration growth," Supercond. Sci. Technol., vol. 28, no. 7, 2015, Art. ID 075009.

[11] M. D. Ainslie et al., "Numerical modelling of iron-pnictide bulk superconductor magnetization," Supercond. Sci. Technol., vol. 30, no. 10, 2017, Art. ID 105009.

[12] C. J. G. Plummer and J. E. Evetts, "Dependence of the shape of the resistive transition on composite inhomogeneity in multifilamentary wires," IEEE Trans. Magn., vol. 23, no. 2, pp. 1179-1182, Mar. 1987.

[13] J. Rhyner, "Magnetic properties and AC-losses of superconductors with power law current-voltage characteristics," Physica C, vol. 212, no. 3-4, pp. 292-300, Jul. 1993.

[14] H. Yamasaki and Y. Mawatari, "Current-voltage characteristics and flux creep in melt-textured $\mathrm{YBa}_{2} \mathrm{Cu}_{3} \mathrm{O}_{7-\delta}$," Supercond. Sci. Technol., vol. 13, pp. 202-208, 2000. 